\title{
Molecular cytogenetics of Dictyoloma vandellianum A. Juss. and the ancestral karyotype of Rutaceae
}

\author{
Amanda Santos ${ }^{1}$ (i), Ana Emília Barros e Silva ${ }^{2}$ (1), Milton Groppo ${ }^{3}$ (i) and Marcelo Guerra ${ }^{1 *}$ (1)
}

Received: October 16, 2020

Accepted: March 9, 2021

\begin{abstract}
Determination of the chromosome base number of a taxon is fundamental to understanding karyotypic variation and its implications for the evolution of that group. This usually requires careful evaluation of cytological literature and robust phylogenetic support. The base number for the family Rutaceae $(x=9$ or $x=18)$ has long been the subject of debate. Here, we analyzed the banding pattern, rDNA sites, and genome size of Dictyoloma vandellianum, subfamily Cneoroideae, the sister group of the remaining Rutaceae, and revised critical points about the chromosome base number of the family. We found that this species has $n=9$, which differs from the $n=18$ possessed by other cytologically known Cneoroideae species. Thus, $n=9$ occurs in the main clades of Rutaceae and is the most probable base number of the family. The hypothesis of $x=18$ as the base number is no longer sustainable, although $n=18$ is very common in Rutaceae. Moreover, the fluorescent banding pattern and the relatively large genome size $(1 \mathrm{C}=$ $1.3 \mathrm{pg}$ ) of $D$. vandellianum suggest that its chromosomal organization is highly divergent from Aurantieae, the only large Rutaceae clade where species with $n=9$ are greatly dominant.
\end{abstract}

Keywords: Aurantieae, chromosome base number, Cneoroideae, Dictyoloma vandelianum, genome size, heterochromatin, rDNA sites

\section{Introduction}

The Rutaceae is a highly diversified and nearly cosmopolitan plant family comprising 150-162 genera and 1500-2096 species (Groppo et al. 2012). The systematic treatment of Engler (1931) split Rutaceae into seven subfamilies, based mainly on fruit and floral characters, although molecular phylogenetic studies suggest the exclusion of the monogeneric subfamily Rhabdodendroideae and the inclusion of some genera from other families (Harrisonia (Simaroubaceae), Cneorum (Cneoraceae), and Ptaeroxylon (Ptaeroxylaceae)) to ensure its monophyletic status (Chase et al. 1999; Groppo et al. 2008; Morton \& Telmer 2014). That infrafamilial division appears unacceptable, however, as no other subfamily having more than one genus is monophyletic (except the subfamily Aurantioideae (Chase et al. 1999), which includes Citrus and related genera).

Currently, only two subfamilies of Rutaceae are recognized (Groppo et al. 2012): Cneoroideae, comprising only eight genera (Appelhans et al. 2011), including Cneorum and Dictyoloma; and Rutoideae, which congregates four former Englerian subfamilies (Rutoideae, Aurantioideae, Toddalioideae, and Flindersioideae). Because of the large economic, agricultural, and ethnobotanical interest in Citrus

1 Laboratório de Citogenética e Evolução de Plantas, Departamento de Botânica, Centro de Biociências, Universidade Federal de Pernambuco, 50670-901, Recife, PE, Brazil

2 Laboratório de Citogenética de Plantas, Departamento de Biociências, Centro de Ciências Agrárias, Universidade Federal da Paraíba, 58397-000, Areia, PB, Brazil

3 Departamento de Biologia, Faculdade de Filosofia, Ciências e Letras de Ribeirão Preto, Universidade de São Paulo, 14051-901, Ribeirão Preto, SP, Brazil

* Corresponding author: msfguerra@hotmail.com 
species, Aurantieae (formerly subfamily Aurantioideae) was, for many years, the only tribe extensively investigated (e.g., Groppo et al. 2012; Wu et al. 2018; Nagano et al. 2018). The karyology and cytotaxonomy of Aurantieae were wellexplored by both classical (Guerra 2009) and molecular methods (Wu et al. 2018; Costa Silva et al. 2019; He et al. 2020; Mendes et al. 2020), although the ancestral or base chromosome number of the family is still a matter of debate (Shan et al. 2006).

It became clear at the beginning of the last century that Citrus and related genera shared the stable chromosome number $n=9$, except for a few polyploids (Guerra et al. 2000; Mou \& Zhang 2012). Smith-White (1954) analyzed 69 Australian Rutaceae species and concluded that the base number of the family was $x=9$, although polyploids with $n=18$, or multiples of 18 , were widespread in the family. A critical genus for his assumption was the highly variable Boronia, with species having $n=7,8,9,11,16,18$, as well as some intraspecific polyploids (later, this range was extended with new counts of $n=10,22,36,54$; see Shan et al. 2006). James (1981) argued, however, that species and genera of Rutaceae with $n=18$ were diploids and suggested that the base number of the family was $x=18$, with the lower numbers representing descending dysploidies. Weston et al. (1984) constructed a cladogram for the species based on 32 morphological traits of 37 species of Boronia, with their known chromosome numbers superimposed, and concluded that $x=9$ explained the chromosome number variation observed in this genus more parsimoniously than $x=18$. Stace et al. (1993) reevaluated that cladogram and noted a progressive reduction from $n=18$ in the most basal lineage (Boronia section Cyanothamnus) to $n=11,9,8$, and 7 in the most derived ones, thus supporting $x=18$ for the genus. Additionally, $n=18$ and $n=36$ were largely dominant among the most basal representatives of the family, suggesting $x$ $=18$ as the ancestral chromosome number for the family as a whole (Stace et al. 1993). The assumption that haploid numbers lower than 18 originated through descending dysploidy was also supported by a comparative analysis of karyotype symmetry in Boronia (Shan et al. 2003a; 2006).

The concept of base (or ancestral) chromosome numbers is currently understood as the haploid number observed in a monophyletic group that most parsimoniously explains the chromosomal variability in that clade, and that shows a clear relationship with the base numbers of the most closely related taxa (Guerra 2000). The correct identification of the base number of a taxon is fundamental to understanding the karyotype variation occurring in that taxon as well as the implications of that variability to the evolution of the group. The search for that number usually demands a careful and critical evaluation of the cytological literature that will eliminate erroneous data and secure robust phylogenetic support (Guerra 2000). Alternatively, the base number can be inferred using probabilistic models (e.g., Freyman \& Höhna 2017), although such methods must be considered with caution as chromosome numbers are controlled by natural selection (Levin 2002). Several important taxonomical rearrangements have been undertaken with Rutaceae, making it necessary to reevaluate the base number of the family in light of current classifications. The most recent classifications of Rutaceae (Groppo et al. 2008, 2012; Morton \& Telmer 2014) recognized a small subfamily, Cneoroideae, as the sister group of all other Rutaceae. The only species of Cneoroideae karyologically know are Cneorum pulverulentum and C. tricocum, both with $2 n=36$ (Chromosome Count Database, Rice et al. 2015; http://ccdb.tau.ac.il/), reinforcing the hypothesis of $\mathrm{x}=18$ for the family.

Karyotype characteristics other than chromosome number, such as genome size, heterochromatic bands, and rDNA chromosomal sites can also be used to better understand karyotype variations and evolution (reviewed by Weiss-Schneeweiss \& Schneeweiss 2013). Heterochromatic bands and several DNA sequence sites have recently been examined in attempts to better understand the chromosome evolution of the former subfamily Aurantioideae (Wu et al. 2018; Yuan et al. 2019; Costa Silva et al. 2019; He et al. 2020; Mendes et al. 2020), which is now included in the subfamily Rutoideae as the tribe Aurantieae - although very little is currently known about other Rutaceae (Shan et al. 2003b).

We present here a detailed karyotypic analysis of Dictyoloma vandellianum, the only representative of the genus (Groppo 2010) formerly placed in the Rutaceae subfamily Dictyolomatoideae but now recognized for the subfamily Cneoroideae. We analyzed its chromosome number and size, the chromosomal locations of heterochromatin and rDNA sites, as well as genome size - and the implications of these characters to understanding the base number of the family are discussed.

\section{Materials and methods}

\section{Plant material}

Two young individuals of Dictyoloma vandellianum A. Juss. were obtained from a seedling nursery in Tambaú, São Paulo, Brazil, and cultivated in the Jardim Experimental do Departamento de Botânica da Universidade Federal de Pernambuco, Recife, Brazil. A voucher was deposited in the Prof. Jayme Coelho de Moraes herbarium (at the Universidade Federal da Paraíba, Brazil, voucher EAN 29425).

\section{Preparation of slides}

For mitotic analyses, young root tips were pretreated with $0.002 \mathrm{M} 8$-hydroxyquinoline at $10^{\circ} \mathrm{C}$ for $24 \mathrm{~h}$, fixed in ethanol-acetic acid (3:1, v/v) for $2 \mathrm{~h}$ at room temperature, and stored at $-20^{\circ} \mathrm{C}$. The fixed root tips were washed in distilled water, digested in a $2 \%$ cellulase (Onozuka)-20\% pectinase 
(Sigma) solution at $37^{\circ} \mathrm{C}$ for one hour, and macerated in a drop of $45 \%$ acetic acid. After coverslip removal in liquid nitrogen, the slides were air-dried and stained with a $2 \mu \mathrm{g} /$ ml DAPI-glycerol (1:1) solution. The best preparations were then selected and subsequently destained in ethanol-acetic acid (3:1), air dried, and aged for 3 days at room temperature.

\section{Chromosome staining with CMA/DAPI and FISH}

Chromosome double staining with the fluorochromes chromomycin A3 (CMA, Sigma) and 4',6-diamidino-2phenylindole (DAPI, Sigma) was performed as described by Moraes et al. (2007). Briefly, the aged slides were stained with CMA $(0.1 \mathrm{mg} / \mathrm{ml})$ for $1 \mathrm{~h}$ followed by DAPI $(1 \mu \mathrm{g} / \mathrm{ml})$ for $30 \mathrm{~min}$, and then mounted in 1:1 (v/v) McIlvaine's $\mathrm{pH}$ 7 buffer-glycerol. Images of the best cells were acquired using a Leica DMLB microscope equipped with a Cohu CCD video camera, and processed using QFISH Leica software.

The fluorescent in situ hybridization (FISH) procedure followed the protocol described by Moraes et al. (2007). The probes used for 5S and 35S rDNA sites were D2 from Lotus japonicas (Regel) K. Larsen and R2 from Arabidopsis thaliana (L.) Heynh., respectively. D2 was labeled with Cy3 dUTP, and the R2 probe was labeled with digoxigenin-11dUTP (Roche), both by nick translation (Invitrogen). The hybridization mixture contained $60 \%$ formamide (v/v), $5 \%$ dextran sulfate $(\mathrm{w} / \mathrm{v}), 2 \times \mathrm{SSC}$, and $2-5 \mathrm{ng} / \mu \mathrm{l}$ of the $35 \mathrm{~S}$ rDNA probe. The $35 \mathrm{~S}$ rDNA probe was detected with sheep anti-digoxygenin-FITC (Roche) antibody, and the signal amplified with FITC-conjugated anti-sheep secondary antibody (Serotec). All preparations were counterstained and mounted with $2 \mu \mathrm{g} / \mathrm{ml}$ DAPI in Vectashield (Vector). Images of cells previously stained with CMA/DAPI were captured as before, and processed for brightness and contrast using Adobe Photoshop CS6.

\section{DNA content estimation and chromosome measurements}

DNA content estimations were performed using a CyFlow SL (Partec) cytometer and Flomax software (Partec), following Doležel et al. (2007). Briefly, small pieces of fresh young leaves from Dictyoloma vandellianum (and from Glycine max (L.) Merr. "Polanka" ( $2 \mathrm{C}=2.55 \mathrm{pg}$ ), as internal standard), were mixed in a glass Petri dish with $0.6 \mathrm{~mL}$ ice-cold Otto buffer I ( $0.1 \mathrm{M}$ citric acid $+0.5 \%$ Tween 20$)$, co-chopped with a razor blade, and filtered through a 30 $\mu \mathrm{m}$ nylon mesh membrane into a cytometry tube. After addition of $0.6 \mathrm{~mL}$ Otto II buffer $\left(0.4 \mathrm{M} \mathrm{Na}_{2} \mathrm{HPO}_{4} 12 \mathrm{H}_{2} \mathrm{O}\right)$ supplemented with RNAse $(50 \mu \mathrm{g} / \mathrm{mL})$ and propidium iodide $(50 \mu \mathrm{g} / \mathrm{mL})$, the samples were immediately analyzed by flow cytometry. Nuclear DNA content ( $2 \mathrm{C}$ value) was calculated as: (sample G1 peak mean/standard G1 peak mean) $\times 2 \mathrm{C}$ DNA content of standard (pg).

In order to evaluate chromosome size and morphology, five metaphases of Dictyoloma vandellianum were measured using Adobe Photoshop CS3 version 10.0 software. The chromosome arm ratio (AR = length of the long arm/length of the short arm) was used to classify the chromosomes as metacentric ( $\mathrm{AR}=1.0-1.4)$, submetacentric $(\mathrm{AR}=1.5$ 2.9), or acrocentric ( $A R \geq 3.0$ ), according to Guerra (1986). Chromosome pairs were ordered from I to VI according to the sizes of their short arms.

\section{Results}

The two plants investigated had $2 n=18$, with metacentric to acrocentric chromosomes, four of them with a terminal secondary constriction and a small satellite (only visible in prometaphase). The CMA/DAPI stained chromosomes displayed two pairs of terminal $\mathrm{CMA}^{+}$bands (Fig. $1 \mathrm{~A}-\mathrm{C}$ ); weak terminal $\mathrm{DAPI}^{+}$bands were sometimes observed in prometaphase chromosomes. The weak $\mathrm{DAPI}^{+}$bands became very well contrasted after in situ hybridization, showing a single terminal DAPI ${ }^{+}$band per chromosome (except with the second largest chromosome pair, which had a DAPI band at both termini) (Fig. 1D). One or two pairs of interstitial dot-like bands were also observed (arrows in Fig. 1D). The rDNA probes revealed two pairs of terminal 35S rDNA sites co-localized with the $\mathrm{CMA}^{+}$bands (Fig. 1E). The only 5S rDNA site observed was adjacent to the 35S rDNA site of the largest chromosome pair, in a subterminal position (Fig. 1F).

The DNA content estimation made using young leaves was $1 C=1.30 \mathrm{pg}$, and the average haploid karyotype length was $32.6 \mu \mathrm{m}$, with individual metaphase chromosomes varying in size from 2.5 to $5.2 \mu \mathrm{m}$. Figure $1 \mathrm{G}$ presents a karyogram based on Figure 1A. The number below each chromosome pair indicates its average length (obtained from five well-spread metaphases). The chromosomes were ordered according to their sizes. They were recognized by the following combination of markers: I - the largest pair with $\mathrm{CMA}^{+}$bands, $5 \mathrm{~S}$ and $35 \mathrm{~S}$ rDNA; II - largest submetacentric pair with a DAPI ${ }^{+}$band at both chromosome termini; III - largest acrocentric; IV - the smallest chromosome pair bearing $\mathrm{CMA}^{+}$bands and $35 \mathrm{~S}$ rDNA sites; $\mathrm{V}$ - chromosomes with two closely located DAPI ${ }^{+}$bands, observed after FISH; VI, second largest acrocentric; VII, VIII, and IX chromosomes without bands, distinguished only by small differences in their sizes.

\section{Discussion}

\section{Comparing the karyotype of Dictyoloma vandellianum with those of Aurantieae}

There is very few information available concerning the karyotypes of other non-Aurantieae Rutaceae having $2 n=18$ beyond their chromosome numbers. The species of Boronia with $2 n=18$ had chromosomes smaller than Dictyoloma 
vandellianum, and there were only meta- to submetacentric chromosomes, except for a single acrocentric pair in $B$. denticulata (Shan et al. 2003a). That latter species had two pairs of 35S rDNA sites, but no 5S rDNA sites (Shan et al. 2003b). No other structural chromosome information for species with $\mathrm{n}=9$ is available, besides for Aurantieae. In relation to Aurantieae, the karyotype of Dictyoloma vandellianum differed in the following aspects: 1 . It had acro/ telocentric chromosomes that are absent in Aurantoideae species (Guerra 1993; Costa Silva et al. 2019); 2. Apart from the $\mathrm{CMA}^{+}$heterochromatic bands associated with $35 \mathrm{~S}$
rDNA sites, Dictyoloma vandelianum had no other $\mathrm{CMA}^{+}$ band, whereas most Aurantieae species have several other $\mathrm{CMA}^{+}$bands associated with different repetitive sequences (Barros e Silva et al. 2010; Deng et al. 2019; He et al. 2020); 3. $\mathrm{DAPI}^{+}$heterochromatic bands, as observed in Dictyoloma vandellianum, were absent in Aurantieae (Guerra et al. 2000). Faint DAPI ${ }^{+}$bands were observed after direct CMA/DAPI staining in Dictyoloma but they became more evident after FISH, due to the differential DNA extraction of the FISH procedure (Barros e Silva \& Guerra 2010); 4. The average chromosome size of Dictyoloma vandellianum was nearly
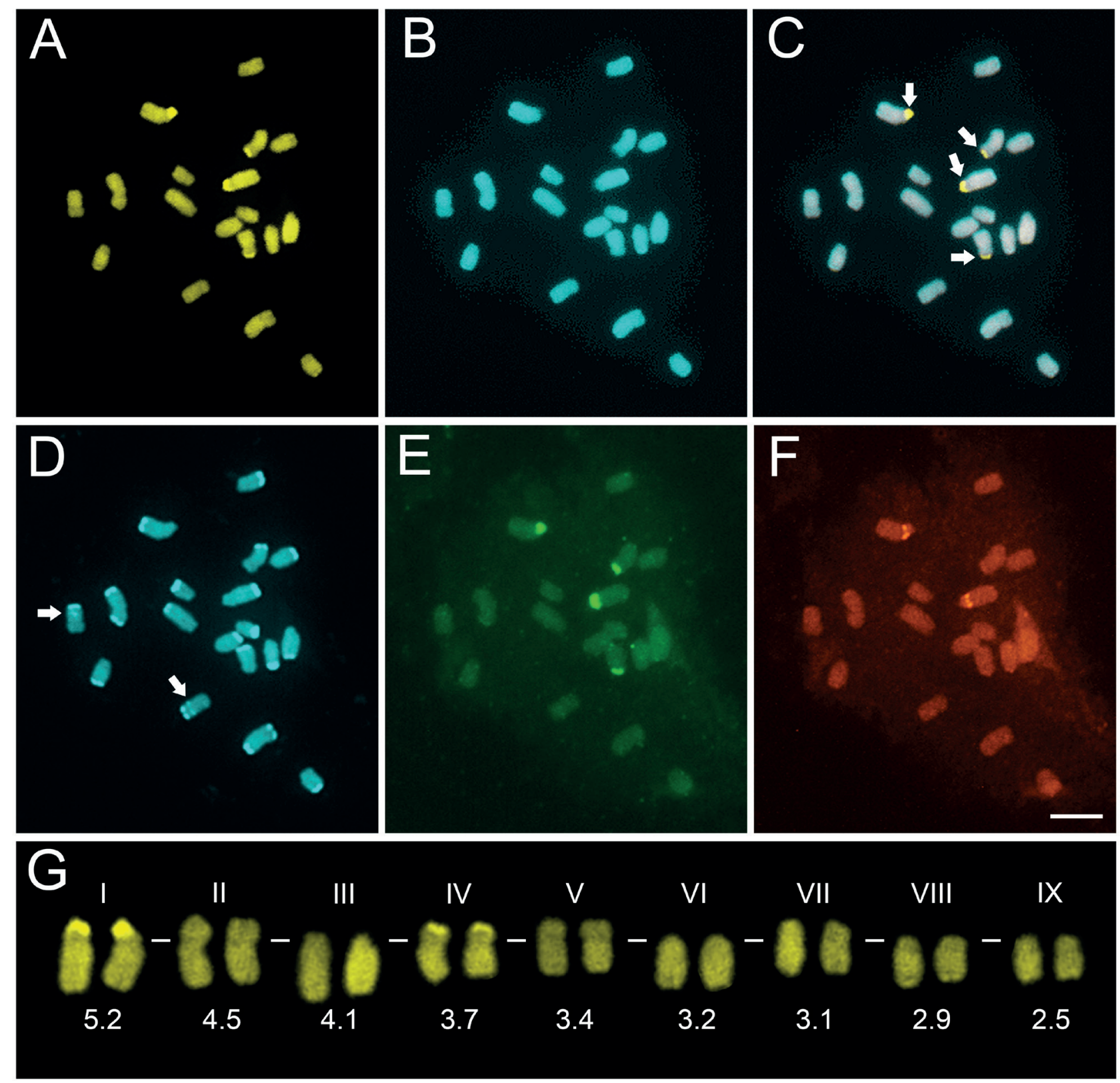

Figure 1. Metaphase of Dictyoloma vandellianum stained in different ways. A-C. CMA (A), DAPI (B), and merged CMA-DAPI image (C). Arrows in $\mathbf{C}$ highlight $\mathrm{CMA}^{+}$bands. E-F. The same cell after FISH showing heterochromatic bands stained with DAPI (D) and 35S (E) and 5S rDNA (F) sites. Arrows in D point to subterminal bands. G. Karyogram showing chromosome pairs ordered from the largest to the smallest and nominated in roman numbers (above). The average chromosome size obtained from five metaphases is indicated below each chromosome pair. Horizontal lines indicate centromeres. Bar in $\mathbf{F}$ corresponds to $5 \mu \mathrm{m}$. 
twice that of Aurantieae (e.g., Costa Silva et al. 2019); 5. The genome size $(1 \mathrm{C}=1.30 \mathrm{pg})$ of Dictyoloma vandellianum was more than twice the average value for Aurantieae species (1C $=0.53 \mathrm{pg}$ ) (The Plant DNA C-values database, release 7.1; https://cvalues.science.kew.org/. Pellicer \& Leitch 2020). Dictyoloma vandellianum has the highest known $1 C$ value among diploid species of Rutaceae, and its average DNA content per chromosome $(0.14 \mathrm{pg})$ is one of the highest for the family (Guerra 1984).

Curiously, Dictyoloma vandellianum displayed a single pair of $5 \mathrm{~S}$ rDNA sites adjacent to one of its 35S rDNA sites, a characteristic observed in nine out of 10 other Aurantieae genera investigated (Barros e Silva et al. 2013). Adjacent $5 \mathrm{~S} / 35 \mathrm{~S}$ rDNA sites have been observed in many angiosperm species, but in no other suprageneric taxon have they been so extensively conserved as in Aurantieae (Roa \& Guerra 2015). It would be interesting to know to what extent this rather instable characteristic is conserved in other Rutaceae. The positions of $35 \mathrm{~S}$ rDNA sites in other Rutaceae species have only been reported for some Boronia species (Shan et al. 2003b), but without information concerning 5 S rDNA sites.

\section{The base number of Cneoroideae and its implication to} the base number of Rutaceae

The finding of $2 n=18$ in Dictyoloma vandellianum (together with the known $2 n=36$ in the closely related species of Cneorum) suggest that the base number of the subfamily Cneoroideae is $x=9$. Assuming Cneoroideae as the sister group to the remaining Rutaceae (Rutoideae or "core Rutaceae"; Groppo et al. 2008; 2012), it can be seen that $n=9$ and $n=18$ are represented in both clades. The core Rutaceae is split into two branches: the tribes Aurantieae and Ruteae (except Dictamnus) plus the genus Chloroxylon and the "RTF Clade", composed of representatives of the former subfamilies Rutoideae (except the genera Ruta and Chloroxylon) and Toddalioideae plus the genus Flindersia (Groppo et al. 2012). Figure 2 indicates the phylogenetic relationships among those main taxa. The haploid numbers $n=9$ and $n=18$ occur in at least one genus of each of these clades, indicating the hypothesis of $x=18$ as unsustainable, as it would imply that a descending dysploid series from $n=18$ to $n=9$ occurred independently in Aurantieae, Cneoroideae, Ruteae, and in the RTF clade.

Within the entire Rutaceae family, the only descending dysploid series known from $n=18$ to $n=9$ (with intermediate numbers $n=10,11,12,16$ ) is that reported by Smith-White (1954) and complemented by Shan et al. (2006) in the genus Boronia (included in the RTF clade). However, the species of the former Boronia section Cyanothamnus, most of them with $n=18$, are now segregated into the genus Cyanothamnus (Duretto et al. 2020). As $n=9$ appears in most sections of Boronia and $n=18$ is now absent, the base number of the genus should be $x=9$ (Weston et al. 1984). The remaining chromosome numbers of Boronia probably arose through dysploidy, polyploidy, and by interspecific hybridizations, as experimentally demonstrated by Astarini et al. (1999).

The base number $x=9$ of Rutaceae appears to be unique within the order Sapindales (Raven 1975), although most other families have been less investigated cytologically. It is currently accepted that all sequenced eudicot genomes are derived from a paleohexaploid ancestor originated through a Whole Genome Triplication that occurred before the split of the rosids and asterids (Jiao et al. 2012; Murat et al. 2017). That was the last polyploidy event before the origin of Citrus and other Aurantieae (Leebens-Mack et al. 2019), and the origin of several triplicated chromosome segments present in the genomes of those species (Xu et al. 2013; Yuan et al. 2019).

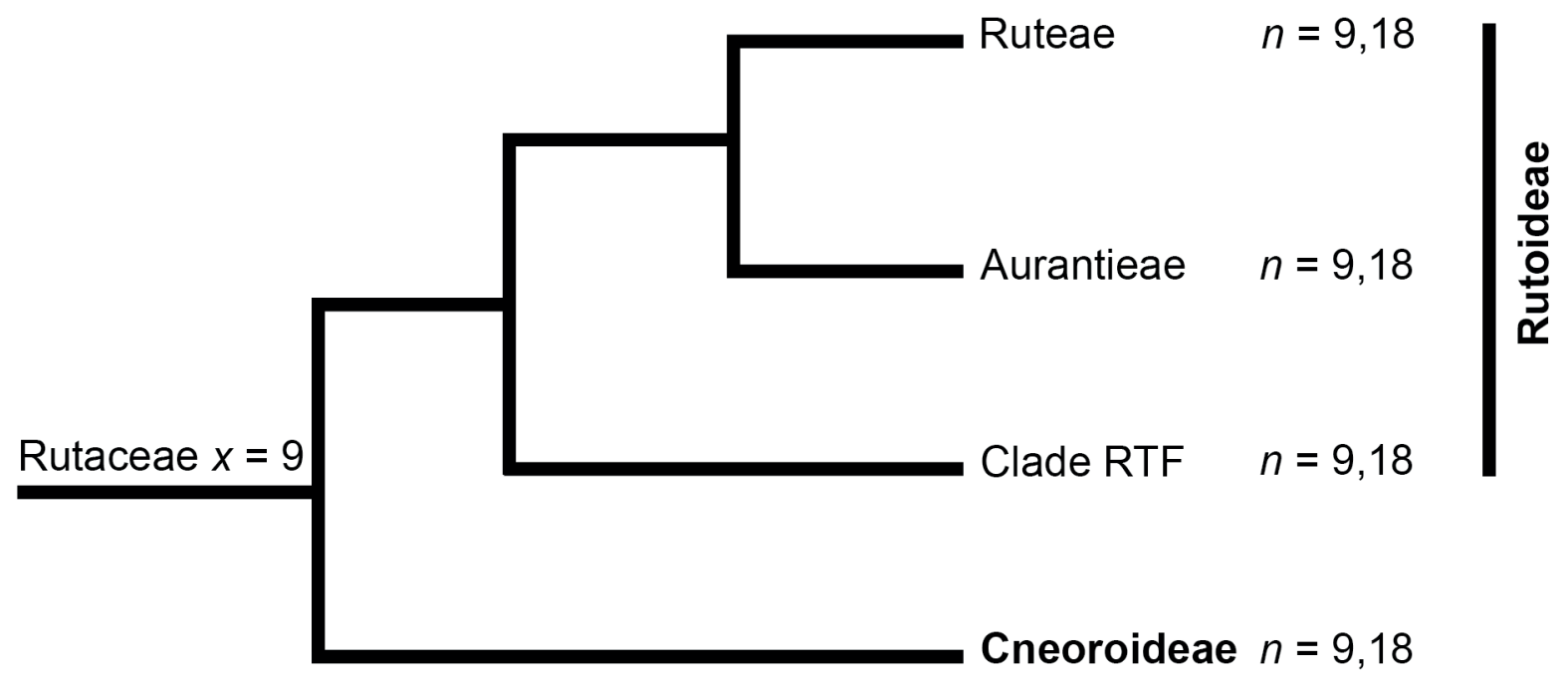

Figure 2. Phylogenetic relationships among Rutaceae main clades. Note the occurrence of chromosome numbers $n=9$ and $n=18$ in all these clades. Subfamilies highlighted in bold. Cladogram modified from Groppo et al. (2012). 
Taken together, our data suggest that the ancestral chromosome number of Rutaceae, $x=9$, has been conserved in some evolutionary lineages since the family originated at 94 Mya and then intensified diversification at 80 Mya (Muellner-Riehl et al. 2016). Nevertheless, structural aspects of the ancestral karyotype (such as chromosome size and morphology, the presence/absence of heterochromatic bands, AT/GC content, nuclear DNA content, among others) are certainly quite distinct among extant $n=9$ lineages. Parallel to the few taxa that conserved $n=9$, other lineages with $n=18$ generated the large number of tetraploid genera found throughout the geographic distribution of the family, such as Zieria, Zanthoxylum, Clausena, Dictamnus, Melicope, Phellodendron, Calodendrum, Esenbeckia, and Flindersia (Stace et al.1993). Additional genome sequencing of other distantly related non-Aurantieae species with $n=9$, such as Dictyoloma vandellianum and some species of Haplophyllum, Thamnosma, and Boronia, would be expected to shed more light on the karyological evolution of Rutaceae.

\section{Acknowledgements}

This research was supported by Conselho Nacional de Desenvolvimento Científico e Tecnológico (CNPq), grant numbers 308903/2011-0, 311924/2016-6 to M. Guerra, and 309088/2016-0 to M. Groppo) and by Fapesp (Fundação de Amparo à Pesquisa do Estado de São Paulo), grants 2006/03170-0, 2007/06336-0, 2016/06260-2 to M. Groppo.

\section{References}

Appelhans MS, Smets E, Razafimandimbison SG, et al. 2011. Phylogeny, evolutionary trends and classification of the Spathelia-Ptaeroxylon clade: morphological and molecular insights. Annals of Botany 107: 1259-1277.

Astarini IA, Yan G, Plummer JA. 1999. Interspecific hybridization of Boronias. Australian Journal of Botany 47: 851-864.

Barros e Silva AE, Guerra M. 2010. The meaning of DAPI bands observed after C-banding and FISH procedures. Biotechnic \& Histochemistry 85: 115-125.

Barros e Silva AE, Marques A, dos Santos KG, Guerra M. 2010. The evolution of CMA bands in Citrus and related genera. Chromosome Research 18: 503-514.

Barros e Silva AE, Soares-Filho WS, Guerra M. 2013. Linked 5S and 45S rDNA sites are highly conserved through the subfamily Aurantioideae (Rutaceae). Cytogenetic and Genome Research 140: 62-69.

Chase MW, Morton CM, Kallunki JA. 1999. Phylogenetic relationships of Rutaceae: a cladistic analysis of the subfamilies using evidence from $\mathrm{rbcL}$ and atpB sequence variation. American Journal of Botany 86: 1191-1199.

Costa Silva S, Mendes S, Regis T, Passos OS, Soares-Filho WS, PedrosaHarand A. 2019. Cytogenetic map of pummelo and chromosome evolution of true citrus species and the hybrid sweet orange. Journal of Agricultural Science 11: 148-160.

Deng HH, Xiang SQ, Guo QG, Jin WW, Cai ZX, Liang GL. 2019. Molecular cytogenetic analysis of genome-specific repetitive elements in Citrus clementina Hort. Ex Tan. and its taxonomic implications. BMC Plant Biology 19: 77. doi: 10.1186/s12870-019-1676-3
Doležel J, Greilhuber J, Suda J. 2007. Estimation of nuclear DNA content in plants using flow cytometry. Nature Protocols 2: 2233-2244.

Duretto MF, Heslewood MM, Bayly MJ. 2020. Boronia (Rutaceae) is polyphyletic: Reinstating Cyanothamnus and the problems associated with inappropriately defined outgroups. Taxon 69: 481-499.

Engler A. 1931. Rutaceae. In: Engler HGA, Prantl K. (eds.) Die natürlichen Pflanzenfamilien. Leipzig, Wilhelm Engelmann. p. 263-266.

Freyman WA, Höhna S. 2017. Cladogenetic and anagenetic models of chromosome number evolution: A Bayesian model averaging approach. Systematic Biology 67: 195-215.

Groppo M, Kallunki JA, Pirani JRR, Antonelli A. 2012. Chilean Pitavia more closely related to Oceania and Old World Rutaceae than Neotropical groups: Evidence from two cpDNA noncoding regions, with a new subfamilial classification of the family. PhytoKeys 19: 9-29.

Groppo M, Pirani JRR, Salatino MLF, Blanco SR, Kallunki JA. 2008. Phylogeny of Rutaceae based on two noncoding regions from cpDNA. American Journal of Botany 95: 985-1005.

Groppo M. 2010. New synonyms in Hortia and Dictyoloma (Rutaceae), with validation of the name Hortia badinii. Novon 20: 163-165.

Guerra M, Santos KGB, Silva A, Ehrendorfer F. 2000. Heterochromatin banding patterns in Rutaceae-Aurantioideae - a case of parallel chromosomal evolution. American Journal of Botany 87: 735-747.

Guerra M. 1984. Cytogenetics of Rutaceae. II. Nuclear DNA content. Caryologia 37: 219-226.

Guerra M. 1986. Reviewing the chromosome nomenclature of Levan et al. Brazilian Journal of Genetics 9: 2-40.

Guerra M. 1993. Cytogenetics of Rutaceae. V. High chromosomal variability in Citrus species revealed by CMA/DAPI staining. Heredity 71: 234-241.

Guerra M. 2000. Chromosome number variation and evolution in monocots. In: Wilson KL, Morrison DA. (eds.) Monocots: Systematics and Evolution. Melbourne, CSIRO Publishing. p. 127-136.

Guerra M. 2009. Chromosomal variability and the origin of Citrus species. In: Mahoney CL, Springer DA. (eds.) Genetic diversity. New York, Nova Science Publishers. p. 51-68.

He L, Zhao H, He J, et al. 2020. Extraordinarily conserved chromosomal synteny of Citrus species revealed by chromosome-specific painting. The Plant Journal 103: 2225-2235.

James SH. 1981. Cytoevolutionary patterns, genetic systems and the phytogeography of Australia. Ecological biogeography of Australia 1: 761-782.

Jiao Y, Wickett NJ, Ayyampalayam S, et al. 2012. Ancestral polyploidy in seed plants and angiosperms. Nature 473: 97-100.

Levin DA. 2002. The role of chromosomal change in plant evolution. New York, Oxford Series in Ecology and Evolution, Oxford University Press.

Mendes S, Regis T, Terol J, Soares Filho SS, Talon M, Pedrosa-Harand A. 2020. Integration of mandarin (Citrus reticulata) cytogenetic map with its genome sequence. Genome 63: 437-444.

Moraes AP, Lemos RR, Brasileiro-Vidal AC, Soares Filho WS, Guerra M. 2007. Chromosomal markers distinguish hybrids and non-hybrid accessions of mandarin. Cytogenetic and Genome Research 119: 275-281.

Morton CM, Telmer C. 2014. New subfamily classification for the Rutaceae. Annals of the Missouri Botanical Garden 99: 620-641.

Mou FJ, Zhang DX. 2012. Chromosome studies in the tribe Clauseneae and the cytological homogeneity in the orange subfamily (Aurantioideae, Rutaceae). Journal of Systematics and Evolution 50: 460-466.

Muellner-Riehl AN, Weeks A, Clayton JW, et al. 2016. Molecular phylogenetics and molecular clock dating of Sapindales based on plastid rbcL, atpB and trnL-trnF DNA sequences. Taxon 65: 10191036.

Murat F, Armero A, Pont C, Klopp C, Salse J. 2017. Reconstructing the genome of the most recent common ancestor of flowering plants. Nature Genetics 49: 490-496.

Nagano Y, Mimura T, Kotoda N, et al. 2018. Phylogenetic relationships of Aurantioideae (Rutaceae) based on RAD-Seq. Tree Genetics \& Genomes 14: 6. doi: 10.1007/s11295-017-1223-z

Leebens-Mack JH, Barker MS, Carpenter EJ, et al. 2019. One thousand plant transcriptomes and the phylogenomics of green plants. Nature 574: 679-685. 
Pellicer J, Leitch IJ. 2020. The Plant DNA C-values database (release 7.1): an updated online repository of plant genome size data for comparative studies. New Phytologist 226: 301-305.

Raven PH. 1975. The bases of angiosperm phylogeny: Cytology. Annals of the Missouri Botanical Garden 62: 724-764.

Rice A, Glick L, Abadi S, et al. 2015. The Chromosome Counts Database (CCDB) - a community resource of plant chromosome numbers. New Phytologist 206: 19-26.

Roa F, Guerra M. 2015. Non-random distribution of 5S rDNA sites and its association with $45 \mathrm{~S}$ rDNA in plant chromosomes. Cytogenetic and Genome Research 146: 243-249.

Shan F, Yan G, Plummer JA. 2003a. Karyotype evolution in the genus Boronia (Rutaceae). Botanical Journal of the Linnean Society 142: 309-320.

Shan F, Yan G, Plummer JA. 2003b. Cytoevolution of Boronia genome revealed by fluorescent in situ hybridisation with $\mathrm{rDNA}$ probes. Genome 46: 507-513.

Shan F, Yan G, Plummer JA. 2006. Basic chromosome number in Boronia (Rutaceae) - competing hypotheses examined. Australian Journal of Botany 54: 681-689.
Smith-White S. 1954. Chromosome numbers in the Boronieae (Rutaceae) and their bearing on the evolutionary development of the tribe in the Australian flora. Australian Journal of Botany 2: 287-303.

Stace HM, Armstrong JA, James SH. 1993. Cytoevolutionary patterns in Rutaceae. Plant Systematics and Evolution 187: 1-28.

Weiss-Schneeweiss H, Schneeweiss GM. 2013. Karyotype diversity and evolutionary trends in angiosperms. In: Greilhuber J, Dolezel J, Wendel J. (eds.) Plant Genome Diversity. Vienna, Springer. p. 209-230.

Weston PH, Carolin RC, Armstrong JA. 1984. A cladistic analysis of Boronia Sm. and Boronella Baill. (Rutaceae). Australian Journal of Botany 32: 187-203.

Wu GA, Terol J, Ibanez V, et al. 2018. Genomics of the origin and evolution of Citrus. Nature 554: 311-316.

Xu Q, Chen LL, Ruan X, et al. 2013. The draft genome of sweet orange (Citrus sinensis). Nature Genetics 45: 59-66.

Yuan J, Wang J, Yu J, et al. 2019. Alignment of Rutaceae genomes reveals lower genome fractionation level than eudicot genomes affected by extra polyploidization. Frontiers in Plant Science 10: 986. doi: 10.3389/fpls.2019.00986 